\title{
Study shows lower vaccination rates for younger siblings after autism spectrum disorder diagnosis in older siblings
}

10.1136/ebnurs-2018-102971

Samantha Vanderslott

Oxford Vaccine Group \& Oxford Martin School, University of Oxford, Oxford, Oxfordshire, UK. Correspondence to: Dr Samantha Vanderslott, Oxford Vaccine Group \& Oxford Martin School, University of Oxford, 34 Broad St, Oxford, OX1 2BD, UK;

samantha.vanderslott@paediatrics.ox.ac.uk

Commentary on: Zerbo 0 , et al. Vaccination patterns in children after autism spectrum disorder diagnosis and in their younger siblings. JAMA Pediatr 2018;172(5):469-75. Implications for practice and research

- The younger siblings of children who received an autism spectrum diagnosis are shown to have lower vaccination rates than the younger siblings of children who have not received an autism spectrum diagnosis. The benefit of applying this knowledge in practice would be to take steps to target this subpopulation.

- The research is novel in that previous studies were not as large or focused. The retrospective matched cohort study uses 'Vaccine Safety Datalink' information from six integrated healthcare delivery systems across the USA.

- Future research could explore strategies for engaging with parents who have received an autism spectrum diagnosis for older children when considering vaccinating younger siblings.

\section{Context}

Much interest has been placed on the reasons for declining vaccination rates and whether the belief that vaccination causes autism plays a part. This study by Zerbo and colleagues 1 asks directly whether such beliefs affect vaccination rates, by exploring whether autism spectrum disorder (ASD) diagnosis in older siblings results in lower vaccination rates in younger siblings. Thus far, as the authors note, there has only been a Canadian study of children with ASD, which found younger siblings were undervaccinated for measles, mumps, and rubella (MMR) and pertussis-containing vaccines compared with children without ASD. 2

\section{Methods}

The study draws from a sample of 592907 children without ASD and 3729 children with ASD (676 (18.1\%) female) and their respective younger siblings. The purpose of the study is to see if the uptake of recommended vaccine doses-according to the 'Advisory Committee on Immunization Practices' (ACIP) for children between the ages of 1 month and 12 years - was lower if an older sibling had received an ASD diagnosis. Parents with a child diagnosed with ASD were more likely to refuse at least one recommended vaccine for a younger sibling and limit the number of vaccines during their first year.

\section{Findings}

As the authors point out, the main implication of the study is the suggestion that children with ASD diagnosis and their younger siblings are at increased risk of vaccine-preventable diseases because they are undervaccinated. The findings suggest that new strategies need to be undertaken to increase vaccine uptake in such subpopulations. The study finds 
statistically significant results that children with ASD were less likely to be fully vaccinated. These findings lead the authors to suggest that current strategies to address vaccine hesitancy are not effective for parents of children with ASD.

\section{Commentary}

The study benefits from a large sample size. It is also a more focused study than similar studies conducted previously because the authors only assess vaccines recommended after diagnosis-and are thus comparing like with like. It would have been helpful to know why the six particular sites were chosen. When the authors list the study's strengths, they point out the large racially/ethnically and socioeconomically diverse population. Was this a criterion for selection of the sites? Also, as the authors point out in their limitations section, it could be interesting to delve deeper and conduct medical record reviews. Further interviews with the families involved (if permitted) would shed light on the reasons why vaccine uptake fell for siblings. The interview number would be manageable given that it is a small group out of a large sample, although it was unclear whether parents would be contactable. An example of a paper that employed this type of methodology involved the authors identifying reports of autism and related disorders on the 'Vaccine Adverse Event Reporting System' (VAERS) and telephoning VAERS reporters to conduct structured interviews about risk perceptions. 3

The authors do not go into detail about the process of how physicians code for vaccine refusal in medical records and why this coding may have been unreliable. Indeed, the grounds for the claim: 'Rates of vaccine refusal in this study likely underestimate the true rates of vaccine refusal because vaccine refusal is not always coded in the medical record consistently by physicians' was not backed up by supporting references or sources. Also, the study refers to previous studies reporting that many parents of children with ASD see vaccines as a contributor to their child's ASD and how such views meant they changed or discontinued vaccination for other children. While there is likely to be a large number of such studies, a reference to an important or illustrative example would have been welcome. Overall, this study helps in understanding falls in vaccine rates and how experiences and beliefs are related, for an important public health concern. Studies such as this one aiming to connect subpopulations with particular actions will become increasingly important in achieving nuanced views of vaccination decisions.

Competing interests None declared.

\section{References}

1. Zerbo O, Modaressi S, Goddard K, et al. Vaccination patterns in children after autism spectrum disorder diagnosis and in their younger siblings. JAMA Pediatr 2018;172:469-75. 2. Abu Kuwaik G, Roberts W, Zwaigenbaum L, et al. Immunization uptake in younger siblings of children with autism spectrum disorder. Autism 2014;18:148-55.

3. Woo EJ, Ball R, Bostrom A, et al. Vaccine risk perception among reporters of autism after vaccination: vaccine adverse event reporting system 1990-2001. Am J Public Health 2004;94:990-5. 